\title{
Analytic Noise-Propagation in Indirect Fan-Beam FBP Reconstruction
}

\author{
Anja Borsdorf ${ }^{1}$, Steffen Kappler ${ }^{2}$, Rainer Raupach ${ }^{2}$, and Joachim Hornegger ${ }^{1}$
}

\begin{abstract}
Precise knowledge of the local image noise is an essential ingredient to efficient application of post-processing methods such as wavelet or diffusion filtering to computed tomography (CT) images. The non-stationary, object dependent nature of noise in $\mathrm{CT}$ images is a direct result from the noise present in the projection data. Since quantum and electronics noise are the dominating noise sources, comparably simple models can be used for direct noise estimates in the individual projections. In this article, we describe the analytic propagation of these noise estimates through fan-beam filtered backprojection (FBP) reconstruction. Contrary to earlier publications in this field, we include the correlations within the parallel projections resulting from the rebinning, the convolution, and the backprojection processes. The method has been validated against Monte-Carlo results and good accuracy with an average relative error below $3.6 \%$ was acchieved for arbitrary objects and over the full range of commonly used convolution kernels and field-of-view settings.
\end{abstract}

\section{INTRODUCTION}

For many applications in Computed Tomography (CT) it would be beneficial having access to a precise estimate of the image noise in each individual pixel. This information can be used for adapting various kinds of post-processing methods to the non-stationary, object dependent nature of noise in CT. In [1], [2] it has been shown that taking into account the local noise variance for wavelet or diffusion filtering of CT reconstructions is essential for getting good results. In addition, several image quality evaluations such as detective quantum efficiency evaluations are based on reliable estimates of the local noise variance in the image. It is possible to get a crude approximation of the local noise variance directly from the reconstructed image or by using two separate reconstructions and noise estimation based on the difference image [1]. These methods have the drawback that the noise estimate needs to be performed within local neighborhoods, making the estimates less reliable on a perpixel basis. A better approach is to use the knowledge about the noise statistics in the projections and analytically propagate it through the reconstruction algorithm. Theoretical descriptions for parallel-beam filtered backprojection can be found e.g. in [3], [4]. In the case of fan-beam FBP reconstruction, image variance has been investigated in [5][8]. These methods are all dealing with direct fan-beam reconstructions without rebinning to parallel projections. In

\footnotetext{
1 A. Borsdorf and J. Hornegger are with with the FriedrichAlexanderUniversity ErlangenNuremberg (FAU), Chair of Pattern Recognition, Martensstr. 3, 91058 Erlangen, Germany (see http://www5.informatik.unierlangen.de).

2 S. Kappler and R. Raupach are with Siemens Healthcare, Siemensstr. 1, 91301 Forchheim, Germany.

The concepts and information presented in this paper is based on research and is not commercially available.
}

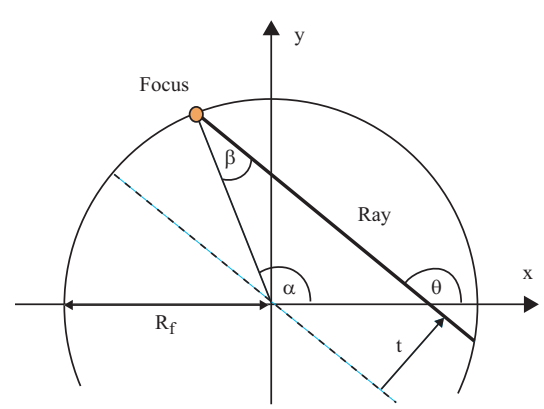

Fig. 1. Geometry and notation used in this paper.

this paper we describe a new approach for propagating noise through indirect fan-beam FBP reconstruction, where the data is first rebinned to parallel-beam geometry. We take into account the correlations induced at the various steps of the reconstruction pipeline. In the evaluation section we experimentally validate the new approach and point out the importance of including the covariances in the computation.

\section{MethoD}

We provide a description of analytic noise propagation through fan-beam reconstruction that is structured as follows:

- Rebinning from fan-beam projections to equidistantly spaced parallel projections.

- Convolution of the rebinned projections with the reconstruction kernel.

- Backprojection of the filtered projections.

- Hounsfield-scaling of reconstructed attenuation coefficients to normalized CT-values.

Instead of reconstructing CT values, an analytical estimate of the error should now be computed for each image pixel. Therefore, every step of the reconstruction pipeline needs to be modified in order to reconstruct noise variances based on the noise estimates in the fan-beam projections. Mainly, all single steps of the reconstruction pipeline (interpolations, convolution and backprojection) can be expressed as linear combinations of noisy data or random variables. For the variance of a linear combination of random variables $X_{i}$ the following holds:

$$
\operatorname{Var}\left(\sum_{i} a_{i} X_{i}\right)=\sum_{i} a_{i}^{2} \operatorname{Var}\left(X_{i}\right)+\sum_{i} \sum_{j \neq i} a_{i} a_{j} \operatorname{Cov}\left(X_{i}, X_{j}\right)
$$

This equation needs to be considered in every single step of the algorithm. Furthermore, the covariance part needs to be modeled properly.

Before the detailed description of the different steps of the noise propagation, we start with a short introduction of the geometry and notation. The basic geometry parameters are 
illustrated in Fig. 1. Given a certain focus angle $\alpha$, the focus position $\left(x_{f}, y_{f}\right)$ is defined as:

$$
x_{f}=R_{f} \cos \alpha, \quad y_{f}=R_{f} \sin \alpha,
$$

where $R_{f}$ is the radius of the focal path. The $\mathrm{x}$-ray path is given by the following equations:

$$
\begin{aligned}
& x_{r}(l)=R_{f} \cos \alpha-l \cos (\alpha+\beta), \\
& y_{r}(l)=R_{f} \sin \alpha-l \sin (\alpha+\beta) .
\end{aligned}
$$

Here, $l \in[0, \infty[$ is the parameter along the ray and $\beta$ describes the fan-angle.

1) Rebinning: The transformation from fan-beam to parallel geometry can be described by the following rebinning equations:

$$
\begin{gathered}
\theta=\alpha+\beta, \\
t=R_{f} \sin \beta .
\end{gathered}
$$

Fig. 1 shows that each ray can be uniquely determined in the $x y$-plane either by $\alpha$ and $\beta$ or by the parallel projection angle $\theta$ and the orthogonal distance $t$ to the isocenter. The resampling from $(\alpha, \beta)$-grid to an equidistantly spaced $(\theta, t)$-grid can be computed differently, using e.g. different interpolation functions. In order to interpolate the noise variances from fan-beam $\left(\operatorname{Var}\left(P_{\alpha}^{\mathrm{fan}}(\beta)\right)\right)$ to parallel geometry $\left(\operatorname{Var}\left(P_{\theta}^{\mathrm{par}}(t)\right)\right)$ basically the following steps are necessary:

- The correlation within the projections needs to be analyzed - For simplicity we assume here that noise in the fan-beam projections is perfectly uncorrelated. This is valid as long as crosstalk at the detector and tube-current variations are negligibly small.

- The weighted sum used for interpolation is replaced by equation (1) - This means that the interpolation weights used during rebinning are replaced by squared weights. If noise in the projections is assumed to be uncorrelated, the covariance part cancels out.

- The correlation induced to the data by the performed interpolation must be modeled - The corresponding interpolation filter, usually a $2 \mathrm{D}$-filter, is computed. The effective filter in $t$ and $\theta$-direction are here approximated as separable linear filters.

In our case, the rebinning is performed in two steps. First, during azimuthal rebinning, equation (5) is applied to obtain a hybrid sinogram depending on the parallel projection angle $\theta$ and the fan angle $\beta$. We use a fixed interpolation grid, keeping the number of grid points constant and perform a linear interpolation in $\alpha$ direction. For interpolating the noise variances the squared interpolation weights need to be used according to equation (1). The azimuthal interpolation introduces no correlations to the hybrid projections in $\beta$ direction. Thus, for the second rebinning step the data can still be assumed to be uncorrelated. Then, the radial rebinning interpolates the hybrid data to an equally distributed set of parallel rays according to equation (6). Here, again a linear interpolation with squared weights is used for interpolating the noise variances. After this interpolation we have the corresponding noise variance $\operatorname{Var}\left(P_{\theta}^{\mathrm{par}}(t)\right)$ for every parallel projection.

After rebinning, noise in the parallel projections and between neighboring views is no longer uncorrelated. The filter function describing azimuthal rebinning is a triangle filter $h^{\text {azi }}(\theta)$. The radial interpolation filter is also modeled as a triangle filter $h^{\mathrm{rad}}(t)$. We approximate the complete interpolation filter as a separable 2D-filter:

$$
h^{\mathrm{ipol}}(\theta, t)=h^{\mathrm{azi}}(\theta) \cdot h^{\mathrm{rad}}(t) .
$$

Therefore, the autocorrelation coefficient function $\rho^{i p o l}(t)$ corresponding to the $2 \mathrm{D}$-interpolation filter is:

$$
\rho^{i p o l}(\theta, t)=h(\theta, t) / h(0,0), \quad h(\theta, t)=h^{\text {ipol }}(\theta, t) * h^{\text {ipol }}(\theta, t) .
$$

2) Convolution: The next step in the reconstruction pipeline is the convolution of the parallel projections $P_{\theta}^{\mathrm{par}}(t)$ with the kernel function $k(t)$ along the row direction $t$ :

$$
P_{\theta}^{\text {con }}(t)=\int P_{\theta}^{\text {par }}\left(t^{\prime}\right) k\left(t-t^{\prime}\right) d t^{\prime} .
$$

In discrete formulation the integral in equation (9) is replaced by a sum. The noise variance in the filtered projections $\operatorname{Var}\left(P_{\theta}^{\text {con }}(t)\right)$ can thus be computed based on equation (1).

Basically, the noise propagation through the convolution can be split into two parts: the convolution with the squared filtering kernel and the consideration of the covariances. The convolution of the noise variances in the parallel projections with the squared filtering kernel has also been considered in the theoretical analysis presented in [4] and [3]. However, our experiments will show, that the covariance terms of the data within the same projections are essential for getting reliable noise estimates. Altogether, the noise variance in the filtered projections can be estimated according to:

$$
\begin{aligned}
& \operatorname{Var}\left(P_{\theta}^{\mathrm{con}}(t)\right)=\sum_{t^{\prime}} \operatorname{Var}\left(P_{\theta}^{\mathrm{par}}\left(t^{\prime}\right)\right) k^{2}\left(t-t^{\prime}\right)+ \\
& \sum_{t^{\prime}} \sum_{t^{\prime \prime} \neq t^{\prime}} \operatorname{Cov}\left(P_{\theta}^{\text {par }}\left(t^{\prime}\right), P_{\theta}^{\text {par }}\left(t^{\prime \prime}\right)\right) k\left(t-t^{\prime}\right) k\left(t-t^{\prime \prime}\right) .
\end{aligned}
$$

The covariance between two channels within one parallel projection can be approximated using the autocorrelation coefficient function in equation (8):

$$
\begin{aligned}
\operatorname{Cov}\left(P_{\theta}^{\text {par }}\right. & \left.\left(t^{\prime}\right), P_{\theta}^{\text {par }}\left(t^{\prime \prime}\right)\right) \approx \\
& \sqrt{\operatorname{Var}\left(P_{\theta}^{\text {par }}\left(t^{\prime}\right)\right) \operatorname{Var}\left(P_{\theta}^{\text {par }}\left(t^{\prime \prime}\right)\right)} \rho^{\text {ipol }}\left(0, t^{\prime}-t^{\prime \prime}\right) .
\end{aligned}
$$

The projections are sampled with the sampling distance $\Delta t$. Consequently, equation (11) can be reformulated such that the distance between $t^{\prime}$ and $t^{\prime \prime}$ in discrete steps of size $\Delta t$ is included:

$$
\begin{gathered}
\operatorname{Var}\left(P_{\theta}^{\mathrm{con}}(t)\right)=\sum_{t^{\prime}} \operatorname{Var}\left(P_{\theta}^{\mathrm{par}}\left(t^{\prime}\right)\right) k^{2}\left(t-t^{\prime}\right)+\sum_{n} \rho^{\mathrm{ipol}}(0, n \Delta t) . \\
\sum_{t^{\prime}}\left\{\sqrt{\operatorname{Var}\left(P_{\theta}^{\text {par }}\left(t^{\prime}\right)\right) \operatorname{Var}\left(P_{\theta}^{\mathrm{par}}\left(t^{\prime}+n \Delta t\right)\right)} k\left(t-t^{\prime}\right) k\left(t-t^{\prime}-n \Delta t\right)+\right. \\
\left.\sqrt{\operatorname{Var}\left(P_{\theta}^{\text {par }}\left(t^{\prime}\right)\right) \operatorname{Var}\left(P_{\theta}^{\text {par }}\left(t^{\prime}-n \Delta t\right)\right)} k\left(t-t^{\prime}\right) k\left(t-t^{\prime}+n \Delta t\right)\right\} .
\end{gathered}
$$

The covariance parts are consequently implemented by additional convolutions. The parameter $n \in\left[1, n_{\max }\right]$ controls the distance between neighboring channels. Usually, the 
autocorrelation function $\rho^{\text {ipol }}(t)$ very rapidly goes to zero. This means that only channels in a small neighborhood are correlated. Therefore, the maximum distance between the channels that need to be considered and thus $n_{\max }$ can be chosen in dependence of the correlation coefficients:

$$
n_{\max }=\min \left\{n \mid\left(\rho^{\mathrm{ipol}}(0, n \Delta t)<\tau\right\} .\right.
$$

Those $n$ for which the correlation coefficient at $n \Delta t$ is below a certain small threshold ${ }^{1} \tau$ are neglected in equation (12).

The convolution process inside the reconstruction pipeline introduces further correlations within the parallel projections. In order to model this for the next step, the autocorrelation coefficient function needs to be computed. The convolution with the kernel function is a linear process. Thus, the correlation inside the data after convolution is described by:

$\rho^{c o n}(\theta, t)=\frac{h(\theta, t)}{h(0,0)}, \quad h(\theta, t)=\mathcal{F}^{-1}\left\{\left(\mathcal{F}\left\{h^{\text {ipol }}(\theta, t) * k(t)\right\}\right)^{2}\right\}$,

where $\mathcal{F}$ denotes the Fourier transformation and $\mathcal{F}^{-1}$ its inverse.

3) Backprojection: After the filtering with the convolution kernel the next step in the reconstruction pipeline is the backprojection into image plane. For each image pixel $\mathbf{x}=$ $(x, y)$ the sum over all $N_{p}$ parallel projection angles $\theta \in$ $[0, \pi[$ is computed:

$$
\mu(\mathbf{x})=\frac{\pi \Delta t}{N_{p}} \sum_{\theta} P_{\theta}^{\operatorname{con}}(x \sin \theta-y \cos \theta) .
$$

Using the definition $P_{\theta, \mathbf{x}}^{\mathrm{con}}:=P_{\theta}^{\operatorname{con}}(x \sin \theta-y \cos \theta)$, the noise variance of the reconstructed attenuation coefficients $\operatorname{Var}(\mu(x, y))$ can be estimated as following:

$$
\begin{aligned}
& \operatorname{Var}(\mu(\mathbf{x}))=\left(\frac{\pi \Delta t}{N_{p}}\right)^{2} \sum_{\theta}\left\{\operatorname{Var}\left(P_{\theta, \mathbf{x}}^{\mathrm{con}}\right)+\right. \\
& \left.\sum_{n=1}^{m}\left(\operatorname{Cov}\left(P_{\theta, \mathbf{x}}^{\mathrm{con}}, P_{\theta-m \Delta \theta, \mathbf{x}}^{\mathrm{con}}\right)+\operatorname{Cov}\left(P_{\theta, \mathbf{x}}^{\mathrm{con}}, P_{\theta+m \Delta \theta, \mathbf{x}}^{\mathrm{con}}\right)\right)\right\} .
\end{aligned}
$$

During the reconstruction algorithm only the azimuthal rebinning introduces a correlation between directly neighboring projections. Thus, only the covariances between neighboring projections are taken into account, meaning $m=1$.

For getting the projection values $P_{\theta}^{\operatorname{con}}(x \sin \theta-y \cos \theta)$ an interpolation is necessary. As already described before, we use here a linear interpolation between neighboring channels. Therefore, the approximation

$$
P_{\theta}^{\mathrm{con}}(x \sin \theta-y \cos \theta) \approx(1-w) P_{\theta}^{\mathrm{con}}\left(t_{l}\right)+w P_{\theta}^{\mathrm{con}}\left(t_{r}\right)
$$

is used during the reconstruction, where $t_{l}<=x \sin \theta-$ $y \cos \theta<=t_{r}$ are the nearest left and right samples and $w \in[0,1]$ defines the linear interpolation weight. The corresponding values in projection $\theta^{\prime}$ are denoted $t_{l}^{\prime}, t_{r}^{\prime}$ and $w^{\prime}$. Consequently, the covariances in eq. (16) amount to:

$$
\begin{gathered}
\operatorname{Cov}\left(P_{\theta, \mathbf{x}}^{\mathrm{con}}, P_{\theta^{\prime}, \mathbf{x}}^{\mathrm{con}}\right)=(1-w)\left(1-w^{\prime}\right) \operatorname{Cov}\left(P_{\theta}^{\mathrm{con}}\left(t_{l}\right), P_{\theta^{\prime}}^{\mathrm{con}}\left(t_{l}^{\prime}\right)\right)+ \\
(1-w) w^{\prime} \operatorname{Cov}\left(P_{\theta}^{\mathrm{con}}\left(t_{l}\right), P_{\theta^{\prime}}^{\mathrm{con}}\left(t_{r}^{\prime}\right)\right)+ \\
w\left(1-w^{\prime}\right) \operatorname{Cov}\left(P_{\theta}^{\mathrm{con}}\left(t_{r}\right), P_{\theta^{\prime}}^{\mathrm{con}}\left(t_{l}^{\prime}\right)\right)+ \\
w w^{\prime} \operatorname{Cov}\left(P_{\theta}^{\mathrm{con}}\left(t_{r}\right), P_{\theta^{\prime}}^{\mathrm{con}}\left(t_{r}^{\prime}\right)\right)
\end{gathered}
$$

\footnotetext{
${ }^{1}$ Typical values for $\tau$ are in the range of 0.01 .
}

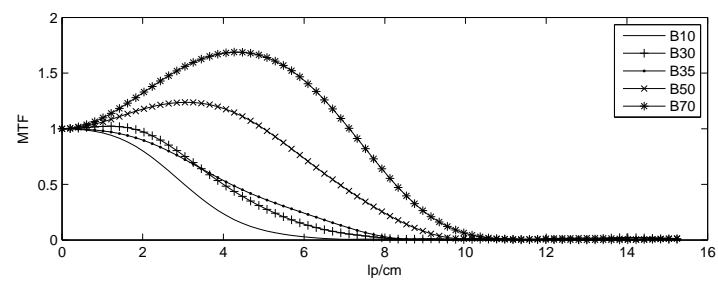

Fig. 2. MTFs of kernels used for the experiments.

and the variance $\operatorname{Var}\left(P_{\theta, \mathbf{x}}^{\text {con }}\right)$ as a special case of eq. (18) results in:

$$
\begin{aligned}
\operatorname{Var}\left(P_{\theta, \mathbf{x}}^{\text {con }}\right)= & (1-w)^{2} \operatorname{Var}\left(P_{\theta}^{\text {con }}\left(t_{l}\right)\right)+w^{2} \operatorname{Var}\left(P_{\theta}^{\text {con }}\left(t_{r}\right)\right)+ \\
& 2(1-w) w \operatorname{Cov}\left(P_{\theta}^{\text {con }}\left(t_{l}\right), P_{\theta}^{\text {con }}\left(t_{r}\right)\right)
\end{aligned}
$$

Furthermore, the covariance between the different samples can be estimated using the autocorrelation coefficient function presented in equation (14):

$$
\begin{aligned}
& \operatorname{Cov}\left(P_{\theta}^{\operatorname{con}}(t), P_{\theta^{\prime}}^{\operatorname{con}}\left(t^{\prime}\right)\right) \approx \\
& \quad \sqrt{\operatorname{Var}\left(P_{\theta}^{\operatorname{con}}(t)\right) \operatorname{Var}\left(P_{\theta^{\prime}}^{\operatorname{con}}\left(t^{\prime}\right)\right)} \rho^{\operatorname{con}}\left(\theta-\theta^{\prime}, t-t^{\prime}\right) .
\end{aligned}
$$

4) Hounsfield-scaling: The reconstructed attenuation coefficients are usually normalized to Hounsfield-Units (HU) according to:

$$
f(\mathbf{x})=\left(\left(\mu(\mathbf{x})-\mu_{w}\right) / \mu_{w}\right) 1000 \mathrm{HU},
$$

with $\mu_{w}$ defining the attenuation coefficient of water. In order to estimate the noise in the normalized reconstructed data, the following equation needs to be used:

$$
\operatorname{Var}(f(\mathbf{x}))=\left(\operatorname{Var}(\mu(\mathbf{x})) / \mu_{w}^{2}\right) 1000^{2} \mathrm{HU}^{2} .
$$

Consequently, the standard deviation of noise $\sigma(\mathbf{x})$ in the reconstructed and normalized image can be computed by

$$
\sigma(\mathbf{x})=\sqrt{\operatorname{Var}(f(\mathbf{x}))}
$$

\section{ACCURRACY OF THE Method}

The analytic model presented in the previous sections makes use of some assumptions and approximations, leading to a systematic error of the method. In addition, the method uses noisy projection data as input to the noise estimation. This leads to an additional intrinsic statistical uncertainty. To quantify systematic and statistical uncertainty, we have carried out Monte-Carlo simulations with the DRASIM software package [9]. We used noise free simulated fanbeam projection data (one full rotation, 1160 projections per $2 \pi, 672$ detector channels per row) of the synthetic phantoms shown in Fig. 3, and built CT-image noise estimates according to the following three procedures:

A) Noise free projections were used for a Poissondistributed noise estimation; propagation through the analytical model yields $\sigma_{A}(\mathbf{x})$.

B) We reconstructed $N=100 \mathrm{CT}$ images, each time adding Poisson-distributed noise to the projections and performed image-based noise measurements from the $N$ images for each pixel: $\sigma_{B}(\mathbf{x})$. 
C) In parallel, for each of the $N$ images we used the noisy projections for Poisson-distributed noise estimation; propagation through the analytical model yields $\sigma_{C}(\mathbf{x})$ and $\operatorname{Var}\left(\sigma_{C}(\mathbf{x})\right)$.

Procedure A) provides the expectation value for the CT image noise according to our method. Measuring the variance of the noise prediction during procedure C) exhibits its intrinsic statistical uncertainty for a given dose and object:

$$
r_{\delta}(\mathbf{x})=\frac{\delta(\mathbf{x})}{\sigma_{A}(\mathbf{x})}, \quad \delta(\mathbf{x})=\sqrt{\operatorname{Var}\left(\sigma_{C}(\mathbf{x})\right)}
$$

For the phantoms under study the relative statistical uncertainties $r_{\delta}(x, y)$ are well below $1 \%$. Subsequently, we use procedure B) to determine the systematic error of our method:

$$
r_{\Delta}(\mathbf{x})=\frac{\Delta(\mathbf{x})}{\sigma_{A}(\mathbf{x})}, \quad \Delta(\mathbf{x})=\sigma_{A}(\mathbf{x})-\sigma_{B}(\mathbf{x})
$$

Table I summarizes the results achieved for the thorax (T) and water (W) phantoms. Examples of the analytic noise propagation results and the corresponding Monte-Carlo simulations are displayed in Fig. 3. The evaluation was carried out for different reconstruction kernels, their modulation transfer functions (MTFs) are shown in Fig. 2. Tab. I lists the average pixel noise values $\bar{\sigma}_{B}$ in $\mathrm{HU}$ for the various kernels as determined by the Monte-Carlo simulations. In addition, the relative systematic errors are listed for three different cases:

1) The covariances in the convolution and backprojection steps are neglected, giving $r_{\Delta, 1}$.

2) The covariances during the backprojection are neglected, giving $r_{\Delta, 2}$.

3) All covariances proposed in our method are taken into account, giving $r_{\Delta, 3}$.

The relative systematic errors are averaged over the whole images and quoted in percent, together with their standard deviations. For the method proposed by us, case number 3 , the range [min., max.] of the relative systematic error occurring in the images is quoted as well. It is evident, that good results over the full range of convolution kernels can only be achieved by considering the covariances during the convolution and backprojection processes. The average relative systematic error is then below $3.6 \%$ for all objects, convolution kernels and field-of-view settings covered by this study.

TABLE I

EVALUATION OF THE SYSTEMATIC ERROR OF THE METHOD PROPOSED. NUMBERS ARE QUOTED IN PERCENT (\%).

\begin{tabular}{|l||ccccc|}
\hline $\mathrm{T}$ & $\mathrm{B} 10$ & $\mathrm{~B} 30$ & $\mathrm{~B} 35$ & $\mathrm{~B} 50$ & $\mathrm{~B} 70$ \\
\hline $\bar{\sigma}_{B}$ & $8.7 \mathrm{HU}$ & $13.6 \mathrm{HU}$ & $13.9 \mathrm{HU}$ & $36.4 \mathrm{HU}$ & $57.6 \mathrm{HU}$ \\
\hline$r_{\Delta, 1}$ & $-92.4 \pm 8.8$ & $-46.1 \pm 10.4$ & $-17.5 \pm 9.8$ & $24.7 \pm 9.1$ & $39.0 \pm 8.1$ \\
$r_{\Delta, 2}$ & $-34.4 \pm 6.2$ & $-26.2 \pm 9.0$ & $-22.1 \pm 10.2$ & $-10.8 \pm 13.3$ & $-6.7 \pm 14.2$ \\
\hline$r_{\Delta, 3}$ & $0.2 \pm 1.6$ & $1.6 \pm 2.0$ & $2.7 \pm 2.5$ & $3.6 \pm 4.1$ & $3.4 \pm 4.8$ \\
range & {$[-6.7,6.6]$} & {$[-4.4,7.6]$} & {$[-4.8,9.6]$} & {$[-9.8,11.7]$} & {$[-12.6,12.5]$} \\
\hline \hline $\mathrm{W}$ & $\mathrm{B} 10$ & $\mathrm{~B} 30$ & $\mathrm{~B} 35$ & $\mathrm{~B} 50$ & $\mathrm{~B} 70$ \\
\hline $\bar{\sigma}_{B}$ & $6.2 \mathrm{HU}$ & $9.9 \mathrm{HU}$ & $10.1 \mathrm{HU}$ & $26.8 \mathrm{HU}$ & $42.6 \mathrm{HU}$ \\
\hline$r_{\Delta, 1}$ & $-94.8 \pm 7.2$ & $-49.5 \pm 9.3$ & $-20.6 \pm 9.1$ & $21.9 \pm 9.2$ & $36.7 \pm 8.5$ \\
$r_{\Delta, 2}$ & $-36.0 \pm 5.1$ & $-29.1 \pm 8.1$ & $-25.3 \pm 9.5$ & $-14.9 \pm 13.5$ & $-10.7 \pm 14.8$ \\
\hline$r_{\Delta, 3}$ & $0.2 \pm 1.6$ & $1.0 \pm 1.9$ & $1.9 \pm 2.5$ & $2.4 \pm 4.2$ & $2.1 \pm 4.9$ \\
range & {$[-6.0,8.8]$} & {$[-5.9,7.6]$} & {$[-5.3,8.7]$} & {$[-10.0,9.7]$} & {$[-13.2,10.1]$} \\
\hline
\end{tabular}

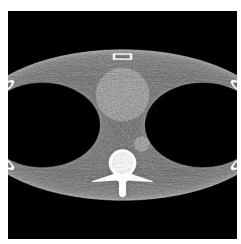

(a) Thorax

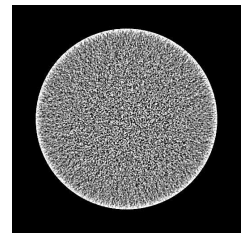

(d) Water

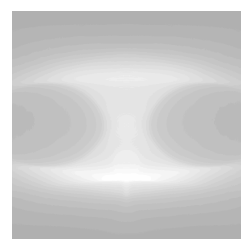

(b) Analytical

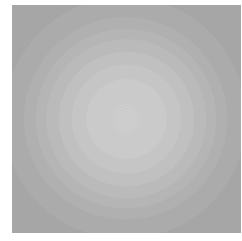

(e) Analytical

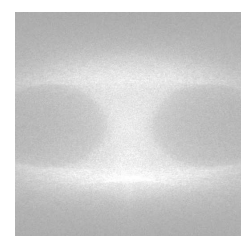

(c) Monte Carlo

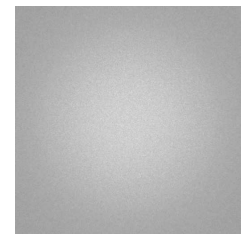

(f) Monte Carlo
Fig. 3. Phantoms used for evaluation reconstructed with B35 (Thorax: $300 \mathrm{~mm}$ FOV, display: $w=50, c=400$, Water: $250 \mathrm{~mm}$ FOV, display: $\mathrm{w}=0, \mathrm{c}=50$ ), together with analytical noise estimates and estimates from 100 noisy realizations (display: $\mathrm{w}=0, \mathrm{c}=50$ ).

\section{CONCLUSIONS}

We proposed a new, fast method for noise-propagation through indirect fan-beam FBP reconstruction with rebinning to parallel-beam geometry. We have developed approximative models for the correlation terms of parallel projection data, resulting from the rebinning and the convolution steps. The method has been validated by Monte-Carlo and demonstrates good accuracy with an average relative error below $3.6 \%$.

\section{ACKNOWLEDGMENT}

The authors gratefully thank Frederic Noo, Adam Wunderlich, Karl Stierstorfer, Heinrich Wallschlaeger, and Dean Ismail-Ibrihem for helpful discussions.

\section{REFERENCES}

[1] A. Borsdorf, et al., "Multiple CT-reconstructions for locally adaptive anisotropic wavelet denoising," International Journal of Computer Assisted Radiology and Surgery, vol. 2, no. 5, pp. 255-264, March 2008.

[2] M. Mayer, et al., "Nonlinear Diffusion vs. Wavelet Based Noise Reduction in CT Using Correlation Analysis," in Vision, Modelling, and Visualisation 2007, Saarbrücken, 2007, pp. 223-232.

[3] A. Kak and M. Slanely, Principles of Computerized Tomographic Imaging. Society for Industrial and Applied Mathematics, July 2001.

[4] T. Buzug, Einführung in die Computertomographie. Berlin Heidelberg: Springer-Verlag, 2004.

[5] X. Pan, "Optimal noise control in and fast reconstruction of fan-beam computed tomography image," Med. Phys., vol. 26, no. 5, pp. 689-697, May 1999.

[6] X. Pan and L. Yu, "Image reconstruction with shift-variant filtration and its implication for noise and resolution properties in fan-beam computed tomography," Med. Phys., vol. 30, no. 4, pp. 590-600, April 2003.

[7] J. Wang, et al., "An alternative solution to the nonuniform noise propagation problem in fan-beam FBP image reconstruction," Med. Phys., vol. 32, no. 11, pp. 3389-3394, November 2005.

[8] A. Wunderlich and F. Noo, "Image covariance and lesion detectability in direct fan-beam x-ray computed tomography," Phys. Med. Biol., vol. 53, no. 10, pp. 2471-2493, April 2008.

[9] K. Stierstorfer, et al., "Segmented multiple plane reconstruction: A novel approximate reconstruction scheme for multi-slice spiral CT," Physics in Medicine and Biology, vol. 47, no. 4, pp. 2571-2581, July 2002. 